\title{
Global existence, energy decay and blow-up of solutions for wave equations with time delay and logarithmic source
}

\section{Sun-Hye Park ${ }^{1 *}$ (D)}

"Correspondence:

sh-park@pusan.ac.kr

1 Office for Education Accreditation,

Pusan National University, Busan,

South Korea

\section{Springer}

\begin{abstract}
In this paper, we study the wave equation with frictional damping, time delay in the velocity, and logarithmic source of the form

$$
u_{t t}(x, t)-\Delta u(x, t)+\alpha u_{t}(x, t)+\beta u_{t}(x, t-\tau)=u(x, t) \ln |u(x, t)|^{\gamma}
$$

There is much literature on wave equations with a polynomial nonlinear source, but not much on the equations with logarithmic source. We show the local and global existence of solutions using Faedo-Galerkin's method and the logarithmic Sobolev inequality. And then we investigate the decay rates and infinite time blow-up for the solutions through the potential well and perturbed energy methods.
\end{abstract}

MSC: 35L05; 35B40; 35B44

Keywords: Wave equation; Logarithmic source; Time delay; Energy decay; Blow-up

\section{Introduction}

We consider the following wave equation with frictional damping, time delay in the velocity, and logarithmic source:

$$
\begin{aligned}
& u_{t t}-\Delta u+\alpha u_{t}(t)+\beta u_{t}(x, t-\tau)=u \ln |u|^{\gamma} \quad \text { for }(x, t) \in \Omega \times(0, \infty), \\
& u(x, t)=0 \quad \text { for }(x, t) \in \partial \Omega \times(0, \infty), \\
& u(x, 0)=u_{0}(x), \quad u_{t}(x, 0)=u_{1}(x) \quad \text { for } x \in \Omega, \\
& u_{t}(x, t)=j_{0}(x, t) \quad \text { for }(x, t) \in \Omega \times(-\tau, 0),
\end{aligned}
$$

where $\Omega \subset \mathbb{R}^{N}, N \geq 1$, is a bounded domain with smooth boundary $\partial \Omega$. $\tau>0$ is time delay, $\alpha, \beta$, and $\gamma$ are real numbers that will be specified later. Equation (1.1) is related to a relativistic version of logarithmic quantum mechanics and many branches of physics such as nuclear physics, optics and geophysics [3, 10, 15].

One of the important theories addressing the existence and nonexistence of solutions for problems with source terms is the potential well method, which was devised by Sattinger

(c) The Author(s) 2020. This article is licensed under a Creative Commons Attribution 4.0 International License, which permits use, sharing, adaptation, distribution and reproduction in any medium or format, as long as you give appropriate credit to the original author(s) and the source, provide a link to the Creative Commons licence, and indicate if changes were made. The images or other third party material in this article are included in the article's Creative Commons licence, unless indicated otherwise in a credit line to the material. If material is not included in the article's Creative Commons licence and your intended use is not permitted by statutory regulation or exceeds the permitted use, you will need to obtain permission directly from the copyright holder. To view a copy of this licence, visit http://creativecommons.org/licenses/by/4.0/. 
[29]. Based on the method, the interaction between the damping and the source terms was firstly considered by Levine [16]. Since then, the damped wave equation with polynomial nonlinear source of the form

$$
u_{t t}-\Delta u+h\left(u_{t}\right)=|u|^{p-2} u
$$

has been studied extensively on existence, nonexistence, stability, and blow-up of solutions (see $[4,12,13,30]$ and the references therein). Recently, much attention has been paid to the study of nonlinear models of hyperbolic and parabolic equations with logarithmic source nonlinearity $[1,2,5-8,17,18,22]$. For the strongly damped wave equation

$$
u_{t t}-\Delta u-a \Delta u_{t}+b u_{t}=u \ln |u|^{\gamma}
$$

Ma and Fang [22] showed the global existence and infinite time blow-up of solutions when $\gamma=2, a=1$, and $b=0$. They used a family of potential wells that is related to the logarithmic nonlinearity, which was introduced by Chen et al. [7]. Lian and $\mathrm{Xu}$ [18] proved the global existence, energy decay and infinite time blow-up of solutions when $\gamma=1$, $a \geq 0$, and $b>-a \lambda$, where $\lambda$ is the first eigenvalue of the operator $-\Delta$ under homogeneous Dirichlet boundary conditions. In [1], the authors considered the plate equation

$$
u_{t t}(x, t)+\Delta^{2} u(x, t)+u(x, t)+u_{t}(x, t)=u(x, t) \ln |u(x, t)|^{\gamma} .
$$

They proved the global existence of solutions and showed that the solutions decay exponentially for a suitable initial data. Later, they extended the results to the case of nonlinear damping in the work [2]. There is not much literature for wave equations with time delay and logarithmic nonlinear source. Thus, in this paper, we intend to study such problem; see (1.1)-(1.4). When $\gamma=0$ in (1.1), Nicaise and Pignotti [24] proved that the energy decays exponentially under the condition $0<\beta<\alpha$, and then improved the result to the case of time varying delay in [25]. For related work on problems with time delay, we also refer to $[9,14,27,31,32]$ and the references therein. Inspired by these results, we discuss the solutions for problem (1.1)-(1.4). To the best of our knowledge, there is little work that takes into account wave equations with time delay and logarithmic source. Thus, we prove the local existence of solutions for problem (1.1)-(1.4) via Faedo-Galerkin's method and the logarithmic Sobolev inequality, and then show the global existence and energy estimates of solutions using the perturbed energy method. Moreover, we establish an infinite time blow-up result by applying the ideas presented in $[20,23,26]$ with some necessary modification.

The outline of this paper is as follows. In Sect. 2, we give some notations and material needed for our work. In Sect. 3, we prove the local existence for problem (1.1)-(1.4). In Sect. 4, we provide the global existence and energy decay rates of solutions. Finally, in Sect. 5 , we show that the solution occurs with an infinite time blow-up.

\section{Preliminaries}

We denote the norm of $X$ by $\|\cdot\|_{X}$ for a Banach space $X$. We denote the scalar product in $L^{2}(\Omega)$ by $(\cdot, \cdot)$. For brevity, we denote $\|\cdot\|_{2}$ by $\|\cdot\|$. Let $B_{1}$ be the optimal constant of the 
embedding inequality

$$
\|u\|^{2} \leq B_{1}\|\nabla u\|^{2} \quad \text { for } u \in H_{0}^{1}(\Omega)
$$

With regard to problem (1.1)-(1.4), we impose the following assumptions:

$\left(H_{1}\right)$ The weights of dissipation and delay satisfy

$$
0<|\beta|<\alpha .
$$

$\left(H_{2}\right)$ The constant $\gamma$ in (1.1) satisfies

$$
0<\gamma<\pi e^{\frac{2(N+1)}{N}} .
$$

Let us list some lemmas for our work.

Lemma 2.1 (Logarithmic Sobolev inequality $[7,11]$ ) For any $u \in H_{0}^{1}(\Omega)$ and any positive real number $k$,

$$
\int_{\Omega} u^{2} \ln |u| d x \leq \frac{1}{2}\|u\|^{2} \ln \|u\|^{2}+\frac{k^{2}}{2 \pi}\|\nabla u\|^{2}-\frac{N}{2}(1+\ln k)\|u\|^{2} .
$$

Remark 2.1 Even though the inequality (2.4) holds for all $k>0$, for the computations throughout this work, we take the constant $k$ satisfying

$$
\rho:=\max \left\{e^{-\frac{N+1}{N}}, \mu^{\frac{1}{N}} \sqrt{\frac{\pi}{\gamma}}\right\}<k<\sqrt{\frac{\pi}{\gamma}},
$$

where $\mu$ is any real number with

$$
0<\mu<1 .
$$

Lemma 2.2 (Logarithmic Gronwall inequality [5]) Let $c>0$ and $l \in L^{1}\left(0, T ; \mathbb{R}^{+}\right)$. If a function $f:[0, T] \rightarrow[1, \infty)$ satisfies

$$
f(t) \leq c\left(1+\int_{0}^{t} l(s) f(s) \ln f(s) d s\right), \quad 0 \leq t \leq T
$$

then

$$
f(t) \leq c e^{c \int_{0}^{t} l(s) d s}, \quad 0 \leq t \leq T .
$$

For $v \in H_{0}^{1}(\Omega)$, we define

$$
\begin{aligned}
& J(v)=\frac{1}{2}\|\nabla v\|^{2}-\frac{1}{2} \int_{\Omega} v^{2}(x) \ln |v(x)|^{\gamma} d x+\frac{\gamma}{4}\|v\|^{2}, \\
& I(v)=\|\nabla v\|^{2}-\int_{\Omega} v^{2}(x) \ln |v(x)|^{\gamma} d x,
\end{aligned}
$$


then

$$
J(v)=\frac{1}{2} I(v)+\frac{\gamma}{4}\|v\|^{2} .
$$

Let

$$
d=\inf _{v \in H_{0}^{1}(\Omega) \backslash\{0\}} \sup _{\lambda \geq 0} J(\lambda v)
$$

then it satisfies, see e.g. [6, 21, 28],

$$
0<d=\inf _{v \in \mathcal{N}} J(v)
$$

where $\mathcal{N}$ is the well-known Nehari manifold, given by

$$
\mathcal{N}=\left\{v \in H_{0}^{1}(\Omega) \backslash\{0\} \mid I(v)=0\right\} .
$$

Lemma 2.3 For any $v \in H_{0}^{1}(\Omega)$ with $\|v\| \neq 0$, the functions I and J satisfy

$$
I(\lambda v)=\lambda \frac{\partial J(\lambda v)}{\partial \lambda} \begin{cases}>0, & 0<\lambda<\lambda^{*}, \\ =0, & \lambda=\lambda^{*}, \\ <0, & \lambda>\lambda^{*},\end{cases}
$$

where

$$
\lambda^{*}=\exp \left(\frac{\|\nabla v\|^{2}-\int_{\Omega} v^{2}(x) \ln |v(x)|^{\gamma} d x}{\gamma\|v\|^{2}}\right) .
$$

Proof By direct computation, we have, for $\lambda \geq 0$,

$$
\begin{aligned}
\lambda \frac{\partial}{\partial \lambda} J(\lambda v)= & \lambda\left\{\lambda\|\nabla v\|^{2}-\lambda \int_{\Omega} v^{2}(x) \ln |v(x)|^{\gamma} d x+\frac{\gamma \lambda}{2}\|v\|^{2}\right. \\
& \left.-\lambda \int_{\Omega} v^{2}(x) \ln |\lambda|^{\gamma} d x-\frac{\gamma \lambda}{2} \int_{\Omega} v^{2}(x) d x\right\} \\
= & \lambda^{2}\left(\|\nabla v\|^{2}-\int_{\Omega} v^{2}(x) \ln |v(x)|^{\gamma} d x-\gamma \ln |\lambda| \int_{\Omega} v^{2}(x) d x\right) \\
= & I(\lambda v),
\end{aligned}
$$

and hence we get the desired result.

Remark 2.2 For a given $v \in H_{0}^{1}(\Omega), J(\lambda v)$ has the absolute maximum value at $\lambda^{*}$, that is,

$$
\sup _{\lambda \geq 0} J(\lambda v)=J\left(\lambda^{*} v\right)=\exp \left(\frac{2\|\nabla v\|^{2}-2 \int_{\Omega} v^{2}(x) \ln |v(x)|^{\gamma} d x}{\gamma\|v\|^{2}}\right) \frac{\gamma}{4}\|v\|^{2} .
$$

Lemma 2.4 The potential depth $d$ in (2.10) satisfies

$$
d \geq \frac{\gamma}{4} e^{N}\left(\frac{\pi}{\gamma}\right)^{\frac{N}{2}}:=E_{1}
$$


Proof From Lemma 2.1, (2.1), and (2.5), we get

$$
\begin{aligned}
I(v) & \geq\left(1-\frac{k^{2} \gamma}{2 \pi}\right)\|\nabla v\|^{2}+\frac{N \gamma}{2}(1+\ln k)\|v\|^{2}-\frac{\gamma}{2}\|v\|^{2} \ln \|v\|^{2} \\
& >\frac{N \gamma}{2}(1+\ln k)\|v\|^{2}-\frac{\gamma}{2}\|v\|^{2} \ln \|v\|^{2} .
\end{aligned}
$$

Taking the limit $k \rightarrow \sqrt{\frac{\pi}{\gamma}}^{-}$, we have

$$
I(v) \geq\left\{\frac{N \gamma}{2}\left(1+\ln \sqrt{\frac{\pi}{\gamma}}\right)-\frac{\gamma}{2} \ln \|v\|^{2}\right\}\|v\|^{2} .
$$

Considering this and (2.12), we have

$$
0=I\left(\lambda^{*} v\right) \geq\left\{\frac{N \gamma}{2}\left(1+\ln \sqrt{\frac{\pi}{\gamma}}\right)-\frac{\gamma}{2} \ln \left\|\lambda^{*} v\right\|^{2}\right\}\left\|\lambda^{*} v\right\|^{2},
$$

and hence

$$
\left\|\lambda^{*} v\right\|^{2} \geq e^{N}\left(\frac{\pi}{\gamma}\right)^{\frac{N}{2}}
$$

Thus, we obtain from (2.13) and (2.9)

$$
\sup _{\lambda \geq 0} J(\lambda v)=J\left(\lambda^{*} v\right)=\frac{1}{2} I\left(\lambda^{*} v\right)+\frac{\gamma}{4}\left\|\lambda^{*} v\right\|^{2}=\frac{\gamma}{4}\left\|\lambda^{*} v\right\|^{2} \geq \frac{\gamma}{4} e^{N}\left(\frac{\pi}{\gamma}\right)^{\frac{N}{2}} .
$$

By the definition of $d$ given in (2.10), we get the desired result.

\section{Local existence of solutions}

In this section we prove the local existence of solutions by applying the ideas in $[1,24]$. Using the function

$$
y(x, \eta, t)=u_{t}(x, t-\eta \tau) \quad \text { for }(x, \eta, t) \in \Omega \times[0,1] \times(0, \infty),
$$

problem (1.1)-(1.4) is rewritten as

$$
\begin{aligned}
& u_{t t}(x, t)-\Delta u(x, t)+\alpha u_{t}(x, t)+\beta y(x, 1, t) \\
& \quad=u(x, t) \ln |u(x, t)|^{\gamma} \quad \text { for }(x, t) \in \Omega \times(0, \infty), \\
& \tau y_{t}(x, \eta, t)+y_{\eta}(x, \eta, t)=0 \quad \text { for }(x, \eta, t) \in \Omega \times(0,1) \times(0, \infty), \\
& u(x, t)=0 \quad \text { for }(x, t) \in \partial \Omega \times(0, \infty), \\
& u(x, 0)=u_{0}(x), \quad u_{t}(x, 0)=u_{1}(x) \quad \text { for } x \in \Omega, \\
& y(x, \eta, 0)=j_{0}(x,-\eta \tau):=y_{0}(x, \eta) \quad \text { for }(x, \eta) \in \Omega \times(0,1) .
\end{aligned}
$$


Definition 3.1 Let $T>0$. We say that $(u, y)$ is a local solution of problem (3.2)-(3.6) if it satisfies the following:

$$
\begin{aligned}
& u \in C\left([0, T] ; H_{0}^{1}(\Omega)\right) \cap C^{1}\left([0, T] ; L^{2}(\Omega)\right) \cap C^{2}\left([0, T) ; H^{-1}(\Omega)\right), \\
& \left(u_{t t}(t), v\right)+(\nabla u(t), \nabla v)+\alpha\left(u_{t}(t), v\right)+\beta(y(1, t), v) \\
& \quad=\left(u(t) \ln |u(t)|^{\gamma}, v\right) \quad \text { for any } v \in H_{0}^{1}(\Omega), \\
& \tau \int_{0}^{1}\left(y_{t}(\eta, t), \varphi(\eta)\right) d \eta+\int_{0}^{1}\left(y_{\eta}(\eta, t), \varphi(\eta)\right) d \eta=0 \quad \text { for any } \varphi \in L^{2}(\Omega \times(0,1)),
\end{aligned}
$$

and

$$
u(0)=u_{0} \quad \text { in } H_{0}^{1}(\Omega), \quad u_{t}(0)=u_{1} \quad \text { in } L^{2}(\Omega), \quad y(0)=y_{0} \quad \text { in } L^{2}(\Omega \times(0,1)) .
$$

Theorem 3.1 Assume that $\left(H_{1}\right)$ and $\left(H_{2}\right)$ hold. Then, for the initial data $u_{0} \in H_{0}^{1}(\Omega), u_{1} \in$ $L^{2}(\Omega), y_{0} \in L^{2}(\Omega \times(0,1))$, there exists a local solution $(u, y)$ of problem $(3.2)-(3.6)$.

Proof Let $\left\{v_{i}\right\}_{i \in \mathbb{N}}$ be orthogonal basis of $H_{0}^{1}(\Omega)$ which is orthonormal in $L^{2}(\Omega)$. Defining $\varphi_{i}(x, 0)=v_{i}(x)$, we can extend $\varphi_{i}(x, 0)$ by $\varphi_{i}(x, \eta)$ over $L^{2}(\Omega \times(0,1))$. We denote $V_{n}=\operatorname{span}\left\{v_{1}, v_{2}, \ldots, v_{n}\right\}$ and $W_{n}=\operatorname{span}\left\{\varphi_{1}, \varphi_{2}, \ldots, \varphi_{n}\right\}$ for $n \geq 1$. We consider the FaedoGalerkin approximation solution $\left(u^{n}, y^{n}\right) \in V_{n} \times W_{n}$ of the form

$$
u^{n}(x, t)=\sum_{i=1}^{n} h_{i}^{n}(t) v_{i}(x) \quad \text { and } \quad y^{n}(x, \eta, t)=\sum_{i=1}^{n} g_{i}^{n}(t) \varphi_{i}(x, \eta), \quad n=1,2, \ldots
$$

solving the approximate system

$$
\begin{aligned}
& \left(u_{t t}^{n}(t), v\right)+\left(\nabla u^{n}(t), \nabla v\right)+\alpha\left(u_{t}^{n}(t), v\right)+\beta\left(y^{n}(1, t), v\right) \\
& \quad=\int_{\Omega} u^{n}(x, t) \ln \left|u^{n}(x, t)\right|^{\gamma} v(x) d x \quad \text { for } v \in V_{n}, \\
& \tau \int_{0}^{1}\left(y_{t}^{n}(\eta, t), \varphi(\eta)\right) d \eta+\int_{0}^{1}\left(y_{\eta}^{n}(\eta, t), \varphi(\eta)\right) d \eta=0 \quad \text { for } \varphi \in W_{n}, \\
& u^{n}(0)=u_{0}^{n}, \quad u_{t}^{n}(0)=u_{1}^{n}, \quad y^{n}(0)=y_{0}^{n},
\end{aligned}
$$

where

$$
u_{0}^{n} \rightarrow u_{0} \quad \text { in } H_{0}^{1}(\Omega), \quad u_{1}^{n} \rightarrow u_{1} \quad \text { in } L^{2}(\Omega), \quad y_{0}^{n} \rightarrow y_{0} \quad \text { in } L^{2}(\Omega \times(0,1)) .
$$

Since problem (3.7)-(3.9) is a normal system of ordinary differential equations, there exists a solution $\left(u^{n}, y^{n}\right)$ on the interval $\left[0, t_{n}\right), t_{n} \in(0, T]$. The extension of this solution to the whole interval $[0, T)$ is a consequence of the estimate below.

Replacing $v$ by $u_{t}^{n}(t)$ in (3.7) and using the relation

$$
\int_{\Omega} u^{n}(x, t) \ln \left|u^{n}(x, t)\right|^{\gamma} u_{t}^{n}(x, t) d x=\frac{d}{d t}\left\{\frac{1}{2} \int_{\Omega}\left(u^{n}(x, t)\right)^{2} \ln \left|u^{n}(x, t)\right|^{\gamma} d x-\frac{\gamma}{4}\left\|u^{n}(t)\right\|^{2}\right\},
$$


we have

$$
\begin{aligned}
\frac{d}{d t} & \left\{\frac{1}{2}\left\|u_{t}^{n}(t)\right\|^{2}+\frac{1}{2}\left\|\nabla u^{n}(t)\right\|^{2}+\frac{\gamma}{4}\left\|u^{n}(t)\right\|^{2}-\frac{1}{2} \int_{\Omega}\left(u^{n}(x, t)\right)^{2} \ln \left|u^{n}(x, t)\right|^{\gamma} d x\right\} \\
& =-\alpha\left\|u_{t}^{n}(t)\right\|^{2}-\beta\left(y^{n}(1, t), u_{t}^{n}(t)\right) .
\end{aligned}
$$

Replacing $\varphi$ by $\omega y^{n}(\eta, t)$ in (3.8), one sees

$$
\frac{\omega \tau}{2} \frac{d}{d t} \int_{\Omega} \int_{0}^{1}\left(y^{n}(x, \eta, t)\right)^{2} d \eta d x=-\frac{\omega}{2}\left\|y^{n}(1, t)\right\|^{2}+\frac{\omega}{2}\left\|y^{n}(0, t)\right\|^{2} .
$$

Collecting (3.10) and (3.11), we get

$$
\frac{d}{d t} E^{n}(t)=-\alpha\left\|u_{t}^{n}(t)\right\|^{2}-\beta\left(y^{n}(1, t), u_{t}^{n}(t)\right)-\frac{\omega}{2}\left\|y^{n}(1, t)\right\|^{2}+\frac{\omega}{2}\left\|y^{n}(0, t)\right\|^{2},
$$

where

$$
\begin{aligned}
E^{n}(t)= & \frac{1}{2}\left\|u_{t}^{n}(t)\right\|^{2}+\frac{1}{2}\left\|\nabla u^{n}(t)\right\|^{2}+\frac{\gamma}{4}\left\|u^{n}(t)\right\|^{2} \\
& -\frac{1}{2} \int_{\Omega}\left(u^{n}(x, t)\right)^{2} \ln \left|u^{n}(x, t)\right|^{\gamma} d x+\frac{\omega \tau}{2}\left\|y^{n}(t)\right\|_{L^{2}(\Omega \times(0,1))}^{2}
\end{aligned}
$$

here

$$
|\beta|<\omega<2 \alpha-|\beta| .
$$

By Young's inequality and the fact $y^{n}(x, 0, t)=u_{t}^{n}(x, t)$, we get

$$
\frac{d}{d t} E^{n}(t) \leq-\left(\alpha-\frac{|\beta|}{2}-\frac{\omega}{2}\right)\left\|u_{t}^{n}(t)\right\|^{2}-\left(\frac{\omega}{2}-\frac{|\beta|}{2}\right)\left\|y^{n}(1, t)\right\|^{2} \leq 0
$$

and

$$
E^{n}(t)+C_{1} \int_{0}^{t}\left\|u_{t}^{n}(s)\right\|^{2} d s+C_{2} \int_{0}^{t}\left\|y^{n}(1, s)\right\|^{2} d s \leq E^{n}(0)
$$

where

$$
C_{1}=\alpha-\frac{|\beta|}{2}-\frac{\omega}{2}>0 \quad \text { and } \quad C_{2}=\frac{\omega}{2}-\frac{|\beta|}{2}>0 .
$$

From this and Lemma 2.1, we observe

$$
\begin{aligned}
& \left\|u_{t}^{n}(t)\right\|^{2}+\left(1-\frac{\gamma k^{2}}{2 \pi}\right)\left\|\nabla u^{n}(t)\right\|^{2}+\frac{\gamma}{2}(1+N(1+\ln k))\left\|u^{n}(t)\right\|^{2} \\
& \quad+2 C_{1} \int_{0}^{t}\left\|u_{t}^{n}(s)\right\|^{2} d s+2 C_{2} \int_{0}^{t}\left\|y^{n}(1, s)\right\|^{2} d s+\omega \tau\left\|y^{n}(t)\right\|_{L^{2}(\Omega \times(0,1))}^{2} \\
& \leq 2 E^{n}(0)+\frac{\gamma}{2}\left\|u^{n}(t)\right\|^{2} \ln \left\|u^{n}(t)\right\|^{2} .
\end{aligned}
$$


Thanks to (2.5), we have

$$
1-\frac{\gamma k^{2}}{2 \pi}>0 \text { and } \frac{\gamma}{2}(1+N(1+\ln k))>0
$$

and hence

$$
\begin{gathered}
\left\|u_{t}^{n}(t)\right\|^{2}+\left\|\nabla u^{n}(t)\right\|^{2}+\left\|u^{n}(t)\right\|^{2}+\int_{0}^{t}\left\|u_{t}^{n}(s)\right\|^{2} d s \\
+\int_{0}^{t}\left\|y^{n}(1, s)\right\|^{2} d s+\left\|y^{n}(t)\right\|_{L^{2}(\Omega \times(0,1))}^{2} \\
\leq c_{1}\left(1+\left\|u^{n}(t)\right\|^{2} \ln \left\|u^{n}(t)\right\|^{2}\right),
\end{gathered}
$$

here and in the sequel $c_{j}, j=1,2, \ldots$, denotes a generic positive constant. On the other hand, it is noted that

$$
u^{n}(x, t)=u^{n}(x, 0)+\int_{0}^{t} u_{t}^{n}(x, s) d s
$$

Applying Cauchy-Schwarz' inequality and (3.17), we get

$$
\begin{aligned}
\left\|u^{n}(t)\right\|^{2} & =2\left\|u^{n}(0)\right\|^{2}+2 T \int_{0}^{t}\left\|u_{t}^{n}(s)\right\|^{2} d s \\
& \leq 2\left\|u^{n}(0)\right\|^{2}+2 T \int_{0}^{t} c_{1}\left(1+\left\|u^{n}(s)\right\|^{2} \ln \left\|u^{n}(s)\right\|^{2}\right) d s \\
& \leq c_{2}\left(1+\int_{0}^{t}\left\|u^{n}(s)\right\|^{2} \ln \left\|u^{n}(s)\right\|^{2} d s\right) .
\end{aligned}
$$

By Lemma 2.2, we find

$$
\left\|u^{n}(t)\right\|^{2} \leq c_{3} e^{c_{4} T}
$$

Since the function $f(s)=s \ln s$ is continuous $(0, \infty), \lim _{s \rightarrow 0^{+}} f(s)=0, \lim _{s \rightarrow+\infty} f(s)=+\infty$, and $f$ decreases on $\left(0, e^{-1}\right)$ and increases on $\left(e^{-1},+\infty\right)$, we have from (3.18) and (3.17)

$$
\begin{gathered}
\left\|u_{t}^{n}(t)\right\|^{2}+\left\|\nabla u^{n}(t)\right\|^{2}+\left\|u^{n}(t)\right\|^{2}+\int_{0}^{t}\left\|u_{t}^{n}(s)\right\|^{2} d s \\
+\int_{0}^{t}\left\|y^{n}(1, s)\right\|^{2} d s+\left\|y^{n}(t)\right\|_{L^{2}(\Omega \times(0,1))}^{2} \leq c_{5} .
\end{gathered}
$$

So, there exists a subsequence of $\left\{\left(u^{n}, y^{n}\right)\right\}$, which we still denote $\left\{\left(u^{n}, y^{n}\right)\right\}$, such that

$$
\begin{aligned}
& u^{n} \rightarrow u \quad \text { weakly star in } L^{\infty}\left(0, T ; H_{0}^{1}(\Omega)\right), \\
& u_{t}^{n} \rightarrow u_{t} \quad \text { weakly star in } L^{\infty}\left(0, T ; L^{2}(\Omega)\right), \\
& y^{n} \rightarrow y \quad \text { weakly star in } L^{\infty}\left(0, T ; L^{2}(\Omega \times(0,1))\right), \\
& y^{n}(1) \rightarrow y(1) \quad \text { weakly in } L^{2}\left(0, T ; L^{2}(\Omega)\right) .
\end{aligned}
$$


By Aubin-Lions' compactness theorem, we find

$$
u^{n} \rightarrow u \quad \text { strongly in } L^{2}\left(0, T ; L^{2}(\Omega)\right)
$$

and

$$
u^{n}(x, t) \rightarrow u(x, t) \quad \text { a.e. in } \Omega \times(0, T)
$$

Since the function $s \rightarrow s \ln |s|^{\gamma}$ is continuous on $\mathbb{R}$,

$$
u^{n}(x, t) \ln \left|u^{n}(x, t)\right|^{\gamma} \rightarrow u(x, t) \ln |u(x, t)|^{\gamma} \quad \text { a.e. in } \Omega \times(0, T) .
$$

Now, we let

$$
\Omega_{1}=\left\{x \in \Omega|| u^{n}(x, t) \mid<1\right\} \quad \text { and } \quad \Omega_{2}=\left\{x \in \Omega|| u^{n}(x, t) \mid \geq 1\right\} .
$$

Then we have

$$
\begin{aligned}
& \int_{\Omega}\left(u^{n}(x, t) \ln \left|u^{n}(x, t)\right|^{\gamma}\right)^{2} d x \\
& =\gamma^{2}\left\{\int_{\Omega_{1}}\left(u^{n}(x, t) \ln \left|u^{n}(x, t)\right|\right)^{2} d x+\int_{\Omega_{2}}\left(u^{n}(x, t) \ln \left|u^{n}(x, t)\right|\right)^{2} d x\right\} \\
& \leq \gamma^{2}\left\{e^{-2}\left|\Omega_{1}\right|+e^{-2}\left(\frac{2}{q-2}\right)^{2} \int_{\Omega_{2}}\left(u^{n}(x, t)\right)^{q} d x\right\} \text { for any } q>2,
\end{aligned}
$$

here we used the fact

$$
|s \ln s| \leq \frac{1}{e} \quad \text { for } 0<s<1 \quad \text { and } \quad s^{-\kappa} \ln s \leq \frac{1}{e \kappa} \quad \text { for } s \geq 1 \text { and } \kappa>0 .
$$

From (3.25) and (3.17), we arrive at

$$
\int_{\Omega}\left(u^{n}(x, t) \ln \left|u^{n}(x, t)\right|^{\gamma}\right)^{2} d x \leq \gamma^{2}\left\{e^{-2}\left|\Omega_{1}\right|+e^{-2}\left(\frac{2}{q-2}\right)^{2} B_{2}^{q}\left\|\nabla u^{n}\right\|^{q}\right\} \leq c_{6},
$$

where $B_{2}$ is the best Sobolev imbedding constant of

$$
H_{0}^{1}(\Omega) \subset L^{q}(\Omega) \quad \text { for } q>2 \text {, if } N=1,2 ; 2<q<\frac{2 N}{N-2} \text {, if } N \geq 3
$$

Thus, we have from (3.26)

$$
u^{n} \ln \left|u^{n}\right|^{\gamma} \text { is uniformly bounded in } L^{\infty}\left(0, T ; L^{2}(\Omega)\right) .
$$

By the Lebesgue bounded convergence theorem, (3.24), and (3.27), we infer

$$
u^{n} \ln \left|u^{n}\right|^{\gamma} \rightarrow u \ln |u|^{\gamma} \quad \text { strongly in } L^{2}\left(0, T ; L^{2}(\Omega)\right) .
$$

Now, we are ready to pass to the limit $m \rightarrow \infty$ in (3.7) and (3.8). The proof of the remainder is standard and can be done as in $[1,19]$. 


\section{Global existence and energy decay estimate}

In this section, we prove the global existence and energy decay rates of solutions to problem (3.2)-(3.6). For this, we define the energy of problem (3.2)-(3.6) as

$$
\begin{aligned}
E(t):= & E(u(t)) \\
= & \frac{1}{2}\left\|u_{t}(t)\right\|^{2}+\frac{1}{2}\|\nabla u(t)\|^{2}+\frac{\gamma}{4}\|u(t)\|^{2} \\
& -\frac{1}{2} \int_{\Omega} u^{2}(x, t) \ln |u(x, t)|^{\gamma} d x+\frac{\omega \tau}{2}\|y(t)\|_{L^{2}(\Omega \times(0,1))}^{2}
\end{aligned}
$$

where $\omega$ is the positive constant given in (3.12). It is noted that

$$
\begin{aligned}
E(t) & =\frac{1}{2}\left\|u_{t}(t)\right\|^{2}+J(u(t))+\frac{\omega \tau}{2}\|y(t)\|_{L^{2}(\Omega \times(0,1))}^{2} \\
& =\frac{1}{2}\left\|u_{t}(t)\right\|^{2}+\frac{1}{2} I(u(t))+\frac{\gamma}{4}\|u(t)\|^{2}+\frac{\omega \tau}{2}\|y(t)\|_{L^{2}(\Omega \times(0,1))}^{2} .
\end{aligned}
$$

By the same arguments as of (3.13), we can deduce

$$
\frac{d}{d t} E(t) \leq-C_{1}\left\|u_{t}(t)\right\|^{2}-C_{2}\|y(1, t)\|^{2} \leq 0
$$

where $C_{1}$ and $C_{2}$ are positive constants given in (3.15).

Lemma 4.1 Assume that $\left(H_{1}\right)$ and $\left(H_{2}\right)$ hold. If $E(0)<d$ and $I\left(u_{0}\right)>0$, then the solution $u$ of problem (1.1)-(1.4) satisfies

$$
I(u(t))>0 \quad \text { for } t \in[0, T)
$$

where $T$ is the maximal existence time of the solutions.

Proof Since $I\left(u_{0}\right)>0$ and $u$ is continuous on $[0, T)$, we know that

$$
I(u(t))>0 \quad \text { for some interval }\left[0, t_{1}\right) \subset[0, T) .
$$

Let $t_{0}$ be the maximum of $t_{1}$ satisfying (4.5). Suppose $t_{0}<T$, then $I\left(u\left(t_{0}\right)\right)=0$, that is,

$$
u\left(t_{0}\right) \in \mathcal{N} .
$$

Thus, we have from (2.11)

$$
J\left(u\left(t_{0}\right)\right) \geq \inf _{v \in \mathcal{N}} J(v)=d .
$$

But this is contradiction to the following relation:

$$
J\left(u\left(t_{0}\right)\right) \leq E\left(t_{0}\right) \leq E(0)<d .
$$

It is noted that $E(t)$ is a nonincreasing positive function from (4.3) and Lemma 4.1. 
Theorem 4.1 Under the conditions of Lemma 4.1, the solution $u$ is global.

Proof It suffices to show that $\left\|u_{t}(t)\right\|^{2}+\|\nabla u(t)\|^{2}$ is bounded independent of $t$. From Lemma 4.1, (4.2), and (4.3), we have

$$
\left\|u_{t}(t)\right\|^{2} \leq\left\|u_{t}(t)\right\|^{2}+I(u(t)) \leq 2 E(t) \leq 2 E(0)<2 d .
$$

Similarly, we see

$$
\|u(t)\|^{2}<\|u(t)\|^{2}+\frac{2}{\gamma} I(u(t))=\frac{4}{\gamma} J(u(t)) \leq \frac{4}{\gamma} E(t) \leq \frac{4}{\gamma} E(0)<\frac{4 d}{\gamma} .
$$

From Lemma 2.1 and (2.8), we infer

$$
\begin{aligned}
\|\nabla u(t)\|^{2} & =I(u(t))+\gamma \int_{\Omega} u^{2}(x, t) \ln |u(x, t)| d x \\
& \leq 2 E(t)+\frac{\gamma}{2}\|u(t)\|^{2} \ln \|u(t)\|^{2}+\frac{k^{2} \gamma}{2 \pi}\|\nabla u(t)\|^{2}-\frac{N \gamma}{2}(1+\ln k)\|u(t)\|^{2} .
\end{aligned}
$$

Taking the limit $k \rightarrow \rho^{+}$in this inequality and using (4.7), we get

$$
\begin{aligned}
\left(1-\frac{\rho^{2} \gamma}{2 \pi}\right)\|\nabla u(t)\|^{2} & \leq 2 E(t)+\frac{\gamma}{2}\left(\ln \|u(t)\|^{2}-N(1+\ln \rho)\right)\|u(t)\|^{2} \\
& <2 d+\frac{\gamma}{2}\left(\ln \left(\frac{4 d}{\gamma}\right)-N(1+\ln \rho)\right)\|u(t)\|^{2} \\
& =2 d+\frac{\gamma}{2}\left\{\ln \left(\frac{4 d}{\gamma} e^{-N} \rho^{-N}\right)\right\}\|u(t)\|^{2}
\end{aligned}
$$

From Lemma 2.4 and (2.5), we get

$$
\ln \left(\frac{4 d}{\gamma} e^{-N} \rho^{-N}\right) \geq \ln \left(\left(\frac{\pi}{\gamma}\right)^{\frac{N}{2}} \rho^{-N}\right)=\ln \left(\left(\sqrt{\frac{\pi}{\gamma}} \rho^{-1}\right)^{N}\right)>\ln 1=0 .
$$

Thus, we observe from (4.8) and (4.7) that

$$
\left(1-\frac{\rho^{2} \gamma}{2 \pi}\right)\|\nabla u(t)\|^{2}<2 d+2 d \ln \left(\frac{4 d}{\gamma} e^{-N} \rho^{-N}\right) .
$$

This gives

$$
\|\nabla u(t)\|^{2}<2 d\left(1-\frac{\rho^{2} \gamma}{2 \pi}\right)^{-1}\left(1+\ln \left(\frac{4 d}{\gamma} e^{-N} \rho^{-N}\right)\right) .
$$

We complete the proof from (4.6) and (4.9).

In order to establish asymptotic behavior for the global solution, let us define the perturbed energy by

$$
\mathcal{E}(t)=E(t)+\varepsilon \Phi(t)+\varepsilon \Xi(t)
$$

where $\varepsilon>0, \Phi(t)=\left(u_{t}(t), u(t)\right)$, and $\Xi(t)=\int_{\Omega} \int_{0}^{1} e^{-\tau \eta} y^{2}(x, \eta, t) d \eta d x$. 
Lemma 4.2 If the conditions of Lemma 4.1 hold, there exist positive constants $C_{3}$ and $C_{4}$ such that

$$
C_{3} E(t) \leq \mathcal{E}(t) \leq C_{4} E(t)
$$

Proof Young's inequality and Lemma 4.1 imply

$$
\begin{aligned}
|\Phi(t)+\Xi(t)| & \leq \frac{1}{2}\left\|u_{t}(t)\right\|^{2}+\frac{1}{2}\|u(t)\|^{2}+\|y(t)\|_{L^{2}(\Omega \times(0,1))}^{2} \\
& \leq \frac{1}{2}\left\|u_{t}(t)\right\|^{2}+\frac{2}{\gamma}\left(\frac{\gamma}{4}\|u(t)\|^{2}+\frac{1}{2} I(u(t))\right)+\|y(t)\|_{L^{2}(\Omega \times(0,1))}^{2} \\
& =\frac{1}{2}\left\|u_{t}(t)\right\|^{2}+\frac{2}{\gamma} J(u(t))+\|y(t)\|_{L^{2}(\Omega \times(0,1))}^{2} \\
& \leq c_{7} E(t) .
\end{aligned}
$$

Taking $\varepsilon>0$ suitably small, we complete the proof.

Theorem 4.2 Let $\left(H_{1}\right)$ and $\left(H_{2}\right)$ hold. Assume that $E(0)<E_{1}$ and $I\left(u_{0}\right)>0$. Then there exist positive constants $C_{0}$ and $C_{5}$ such that

$$
0<E(t) \leq C_{0} e^{-C_{5} t} \quad \text { for } t \geq 0
$$

Proof Using (3.2) and Young's inequality, we have

$$
\begin{aligned}
\Phi^{\prime}(t)= & \left\|u_{t}(t)\right\|^{2}-\|\nabla u(t)\|^{2}-\alpha\left(u_{t}(t), u(t)\right)-\beta(y(1, t), u(t)) \\
& +\int_{\Omega} u^{2}(x, t) \ln |u(x, t)|^{\gamma} d x \\
\leq & \left\|u_{t}(t)\right\|^{2}-\frac{1}{2}\|\nabla u(t)\|^{2}+\alpha^{2} B_{1}\left\|u_{t}(t)\right\|^{2}+\beta^{2} B_{1}\|y(1, t)\|^{2} \\
& +\int_{\Omega} u^{2}(x, t) \ln |u(x, t)|^{\gamma} d x .
\end{aligned}
$$

From (3.3) and the integration by parts, we get

$$
\begin{aligned}
\Xi^{\prime}(t) & =-\frac{2}{\tau} \int_{\Omega} \int_{0}^{1} e^{-\tau \eta} y(x, \eta, t) y_{\eta}(x, \eta, t) d \eta d x \\
& =-\frac{1}{\tau} \int_{\Omega} \int_{0}^{1} e^{-\tau \eta} \frac{\partial}{\partial \eta} y^{2}(x, \eta, t) d \eta d x \\
& =-\frac{e^{-\tau}}{\tau}\|y(1, t)\|^{2}+\frac{1}{\tau}\|y(0, t)\|^{2}-\int_{\Omega} \int_{0}^{1} e^{-\tau \eta} y^{2}(x, \eta, t) d \eta d x \\
& \leq \frac{1}{\tau}\left\|u_{t}(t)\right\|^{2}-e^{-\tau} \int_{\Omega} \int_{0}^{1} y^{2}(x, \eta, t) d \eta d x
\end{aligned}
$$


Collecting these and (4.3), we have

$$
\begin{aligned}
\mathcal{E}^{\prime}(t) \leq & -\left(C_{1}-\varepsilon-\varepsilon \alpha^{2} B_{1}-\frac{\varepsilon}{\tau}\right)\left\|u_{t}(t)\right\|^{2}-\frac{\varepsilon}{2}\|\nabla u(t)\|^{2}-\left(C_{2}-\varepsilon \beta^{2} B_{1}\right)\|y(1, t)\|^{2} \\
& +\varepsilon \int_{\Omega} u^{2}(x, t) \ln |u(x, t)|^{\gamma} d x-\varepsilon e^{-\tau}\|y(t)\|_{L^{2}(\Omega \times(0,1))}^{2}
\end{aligned}
$$

Subtracting and adding $\xi E(t)$ with $0<\xi<2 \varepsilon$, we have

$$
\begin{aligned}
\mathcal{E}^{\prime}(t) \leq & -\xi E(t)-\left(C_{1}-\varepsilon-\varepsilon \alpha^{2} B_{1}-\frac{\varepsilon}{\tau}-\frac{\xi}{2}\right)\left\|u_{t}(t)\right\|^{2}-\left(\frac{\varepsilon}{2}-\frac{\xi}{2}-\frac{\xi \gamma B_{1}}{4}\right)\|\nabla u(t)\|^{2} \\
& -\left(C_{2}-\varepsilon \beta^{2} B_{1}\right)\|y(1, t)\|^{2}+\left(\varepsilon-\frac{\xi}{2}\right) \int_{\Omega} u^{2}(x, t) \ln |u(x, t)|^{\gamma} d x \\
& -\left(\varepsilon e^{-\tau}-\frac{\xi \omega \tau}{2}\right)\|y(t)\|_{L^{2}(\Omega \times(0,1))}^{2}
\end{aligned}
$$

From the logarithmic Sobolev inequality, we obtain

$$
\begin{aligned}
\mathcal{E}^{\prime}(t) \leq & -\xi E(t)-\left(C_{1}-\varepsilon-\varepsilon \alpha^{2} B_{1}-\frac{\varepsilon}{\tau}-\frac{\xi}{2}\right)\left\|u_{t}(t)\right\|^{2} \\
& -\left\{\varepsilon\left(\frac{1}{2}-\frac{\gamma k^{2}}{2 \pi}\right)-\frac{\xi}{2}\left(1-\frac{\gamma k^{2}}{2 \pi}\right)-\frac{\xi \gamma B_{1}}{4}\right\}\|\nabla u(t)\|^{2} \\
& +\frac{\gamma}{2}\left(\varepsilon-\frac{\xi}{2}\right)\left\{\ln \|u(t)\|^{2}-N(1+\ln k)\right\}\|u(t)\|^{2} \\
& -\left(C_{2}-\varepsilon \beta^{2} B_{1}\right)\|y(1, t)\|^{2}-\left(\varepsilon e^{-\tau}-\frac{\xi \omega \tau}{2}\right)\|y(t)\|_{L^{2}(\Omega \times(0,1))}^{2}
\end{aligned}
$$

First, we choose $\varepsilon>0$ small such that

$$
C_{1}-\varepsilon-\varepsilon \alpha^{2} B_{1}-\frac{\varepsilon}{\tau}>0 \text { and } C_{2}-\varepsilon \beta^{2} B_{1}>0
$$

Then, taking $\xi>0$ sufficiently small and noting that $\frac{1}{2}-\frac{\gamma k^{2}}{2 \pi}>0$ (see (2.5)), we arrive at

$$
\mathcal{E}^{\prime}(t) \leq-\xi E(t)+\frac{\gamma}{2}\left(\varepsilon-\frac{\xi}{2}\right)\left\{\ln \|u(t)\|^{2}-N(1+\ln k)\right\}\|u(t)\|^{2}
$$

Since $0<E(0)<E_{1}$, there exists $0<\mu<1$ such that $E(0)=\mu E_{1}$. Thus, we have from (4.7)

$$
\ln \|u(t)\|^{2}<\ln \left(\frac{4}{\gamma} E(t)\right) \leq \ln \left(\frac{4}{\gamma} E(0)\right)=\ln \left(\frac{4 \mu E_{1}}{\gamma}\right)=\ln \left(\mu e^{N}\left(\frac{\pi}{\gamma}\right)^{\frac{N}{2}}\right) .
$$

Thus, we infer from (2.5) that

$$
\begin{aligned}
\ln \|u(t)\|^{2}-N(1+\ln k) & \leq \ln \left(\mu e^{N}\left(\frac{\pi}{\gamma}\right)^{\frac{N}{2}}\right)-N(1+\ln k) \\
& =N \ln \left(\mu^{\frac{1}{N}} \sqrt{\frac{\pi}{\gamma}} k^{-1}\right)<N \ln 1=0 .
\end{aligned}
$$


Substituting this into (4.10), we conclude

$$
\mathcal{E}^{\prime}(t) \leq-\xi E(t)
$$

Consequently, we complete the proof from Lemma 4.2.

\section{Infinite time blow-up}

In this section, inspired by the ideas in $[20,23,26]$, we establish a blow-up result for problem (1.1)-(1.4). For this, we first give the following lemma.

Lemma 5.1 Assume that $\left(H_{1}\right)$ and $\left(H_{2}\right)$ hold. If $E(0)<E_{1}$ and $I\left(u_{0}\right)<0$, then the solution $u$ of problem (1.1)-(1.4) satisfies

$$
I(u(t))<0 \quad \text { for } t \in[0, T)
$$

and

$$
\|u(t)\|^{2}>\frac{4 E_{1}}{\gamma} \quad \text { for } t \in[0, T),
$$

where $T$ is the maximal existence time of solutions.

Proof Since $I\left(u_{0}\right)<0$ and $u$ is continuous on $[0, T)$, we know that

$$
I(u(t))<0 \quad \text { for some interval }\left[0, t_{1}\right) \subset[0, T) .
$$

Let $t_{0}$ be the maximal time satisfying (5.3) and suppose $t_{0}<T$, then $I\left(u\left(t_{0}\right)\right)=0$, that is,

$$
u\left(t_{0}\right) \in \mathcal{N} .
$$

Thus, we have

$$
d \leq J\left(u\left(t_{0}\right)\right)=\frac{1}{2} I\left(u\left(t_{0}\right)\right)+\frac{\gamma}{4}\left\|u\left(t_{0}\right)\right\|^{2} \leq E\left(u\left(t_{0}\right)\right) \leq E(0)<E_{1} .
$$

This is in contradiction to Lemma 2.4. So, (5.1) is proved. From Lemma 2.4, (2.13), and (5.1), we find

$$
\begin{aligned}
E_{1} & \leq d \leq J\left(\lambda^{*} u(t)\right)=\exp \left(\frac{2\|\nabla u(t)\|^{2}-2 \int_{\Omega} u^{2}(x, t) \ln |u(x, t)|^{\gamma} d x}{\gamma\|u(t)\|^{2}}\right) \frac{\gamma}{4}\|u(t)\|^{2} \\
& <\frac{\gamma}{4}\|u(t)\|^{2} .
\end{aligned}
$$

Thus, we complete the proof.

Theorem 5.1 Assume that $\left(H_{1}\right)$ and $\left(H_{2}\right)$ hold. Assume that $E(0)<\zeta E_{1}$, where $0<\zeta<1$, and $I\left(u_{0}\right)<0$. Then the solution of problem (1.1)-(1.4) blows up at infinity. 
Proof We set

$$
F(t)=\zeta E_{1}-E(t)
$$

From (4.3), we have

$$
F^{\prime}(t)=-E^{\prime}(t) \geq C_{1}\left\|u_{t}(t)\right\|^{2}+C_{2}\|y(1, t)\|^{2} \geq 0 .
$$

From (5.5), (4.1), and (5.2), we observe

$$
\begin{aligned}
0 & <F(0) \leq F(t) \leq \zeta E_{1}+\frac{1}{2} \int_{\Omega} u^{2}(x, t) \ln |u(x, t)|^{\gamma} d x \\
& <\frac{\gamma}{4}\|u(t)\|^{2}+\frac{1}{2} \int_{\Omega} u^{2}(x, t) \ln |u(x, t)|^{\gamma} d x .
\end{aligned}
$$

Now, we define

$$
G(t)=F(t)+\varepsilon\left(u(t), u_{t}(t)\right)+\frac{\varepsilon \alpha}{2}\|u(t)\|^{2} .
$$

Using (3.2), (4.1), we have

$$
\begin{aligned}
G^{\prime}(t)= & F^{\prime}(t)+\varepsilon\left\|u_{t}(t)\right\|^{2}-\varepsilon\|\nabla u(t)\|^{2}-\varepsilon \beta(u(t), y(1, t))+\varepsilon \int_{\Omega} u^{2}(x, t) \ln |u(x, t)|^{\gamma} d x \\
= & F^{\prime}(t)+2 \varepsilon\left\|u_{t}(t)\right\|^{2}-\varepsilon \beta(u(t), y(1, t))-2 \varepsilon E(t) \\
& +\frac{\varepsilon \gamma}{2}\|u(t)\|^{2}+\omega \tau\|y(t)\|_{L^{2}(\Omega \times(0,1))}^{2} .
\end{aligned}
$$

By Young's inequality and (5.5), we get

$$
\beta(u(t), y(1, t)) \leq|\beta|\left(\delta\|u(t)\|^{2}+\frac{1}{4 \delta}\|y(1, t)\|^{2}\right) \leq \delta|\beta|\|u(t)\|^{2}+\frac{|\beta|}{4 \delta C_{2}} F^{\prime}(t) .
$$

Adapting this to (5.7) and using (5.4) and (5.2), we get

$$
\begin{aligned}
G^{\prime}(t) \geq & \left(1-\frac{\varepsilon|\beta|}{4 \delta C_{2}}\right) F^{\prime}(t)+2 \varepsilon\left\|u_{t}(t)\right\|^{2}+\left(\frac{\varepsilon \gamma}{2}-\varepsilon|\beta| \delta\right)\|u(t)\|^{2} \\
& +2 \varepsilon F(t)-2 \varepsilon \zeta E_{1}+\omega \tau\|y(t)\|_{L^{2}(\Omega \times(0,1))}^{2} \\
\geq & \left(1-\frac{\varepsilon|\beta|}{4 \delta C_{2}}\right) F^{\prime}(t)+2 \varepsilon\left\|u_{t}(t)\right\|^{2}+\varepsilon\left((1-\zeta) \frac{\gamma}{2}-|\beta| \delta\right)\|u(t)\|^{2} \\
& +2 \varepsilon F(t)+\omega \tau\|y(t)\|_{L^{2}(\Omega \times(0,1))}^{2}
\end{aligned}
$$

First, we fix $\delta>0$ such that $(1-\zeta) \frac{\gamma}{2}-|\beta| \delta>0$, then choose $\varepsilon>0$ sufficiently small so that $1-\frac{\varepsilon|\beta|}{4 \delta C_{2}}>0$. Then we have from (5.5)

$$
G^{\prime}(t) \geq c_{8}\left(F(t)+\left\|u_{t}(t)\right\|^{2}+\|u(t)\|^{2}\right) \geq 0
$$


On the other hand, we can easily see that

$$
G(t) \leq c_{9}\left(F(t)+\left\|u_{t}(t)\right\|^{2}+\|u(t)\|^{2}\right) .
$$

Let us take $\varepsilon>0$ sufficiently small again to get

$$
G(0)=F(0)+\varepsilon\left(u_{0}, u_{1}\right)+\frac{\varepsilon \alpha}{2}\left\|u_{0}\right\|^{2}>0 .
$$

Then we obtain from (5.9) and (5.11)

$$
G(t) \geq G(0)>0 .
$$

From (5.9) and (5.10), we observe

$$
G^{\prime}(t) \geq c_{10} G(t),
$$

and hence

$$
G(t) \geq e^{c_{10} t} G(0)>0 .
$$

Thus, $G(t)$ blows up at infinity.

\section{Acknowledgements}

The author is grateful to the anonymous referees for the careful reading and their important comments to improve this paper.

\section{Funding}

This research was supported by Basic Science Research Program through the National Research Foundation of Korea (NRF) funded by the Ministry of Education (2020R1I1A3066250).

\section{Availability of data and materials}

Not applicable.

\section{Competing interests}

The author declares that they have no competing interests.

\section{Authors' contributions}

The author read and approved the final manuscript.

\section{Publisher's Note}

Springer Nature remains neutral with regard to jurisdictional claims in published maps and institutional affiliations.

Received: 12 June 2020 Accepted: 7 October 2020 Published online: 10 December 2020

\section{References}

1. Al-Gharabli, M.M., Messaoudi, S.A.: The existence and the asymptotic behavior of a plate equation with frictional damping and a logarithmic source term. J. Math. Anal. Appl. 454, 1114-1128 (2017)

2. Al-Gharabli, M.M., Messaoudi, S.A.: Existence and a general decay result for a plate equation with nonlinear damping and a logarithmic source term. J. Evol. Equ. 18, 105-125 (2018)

3. Bialynicki-Birula, I., Mycielski, J.: Nonlinear wave mechanics. Ann. Phys. 100, 62-93 (1976)

4. Cavalcanti, M.M., Domingos Cavalcanti, V.N., Martinez, P.: Existence and decay rate estimates for the wave equation with nonlinear boundary damping and source term. J. Differ. Equ. 203, 119-158 (2004)

5. Cazenave, T., Haraux, A.: Equations d'evolution avec non-linearite logarithmique. Ann. Fac. Sci. Toulouse Math. 2(1), $21-51(1980)$

6. Chen, H., Liu, G.: Global existence and nonexistence for semilinear parabolic equations with conical degeneration. J. Pseudo-Differ. Oper. Appl. 3, 329-349 (2012)

7. Chen, H., Luo, P., Liu, G.: Global solution and blow-up of a semilinear heat equation with logarithmic nonlinearity. J. Math. Anal. Appl. 442, 84-98 (2015) 
8. Chen, H., Tian, S.: Initial boundary value problem for a class of semilinear pseudo-parabolic equations with logarithmic nonlinearity. J. Differ. Equ. 258, 4424-4442 (2015)

9. Datko, R.: Not all feedback stabilized hyperbolic systems are robust with respect to small time delays in their feedbacks. SIAM J. Control Optim. 26, 697-713 (1988)

10. Gorka, P.: Logarithmic Klein-Gordon equation. Acta Phys. Pol. B 40, 59-66 (2009)

11. Gross, L.: Logarithmic Sobolev inequalities. Am. J. Math. 97, 1061-1083 (1975)

12. Ha, T.G.: Blow-up for wave equation with weak boundary damping and source terms. Appl. Math. Lett. 49, 166-172 (2015)

13. Ikehata, R.: Some remarks on the wave equations with nonlinear damping and source terms. Nonlinear Anal. 27, $1165-1175(1996)$

14. Kirane, M., Said-Houari, B.: Existence and asymptotic stability of a viscoelastic wave equation with a delay. Z. Angew. Math. Phys. 62, 1065-1082 (2011)

15. Koutvitsky, V.A., Maslov, E.M.: Instability of coherent states of a real scalar field. J. Math. Phys. 47, Article ID 022302 (2006)

16. Levine, H.A.: Some additional remarks on the nonexistence of global solutions to nonlinear wave equation. SIAM J. Math. Anal. 5, 138-146 (1974)

17. Lian, W., Ahmed, M.S., Xu, R.: Global existence and blow up of solution for semilinear hyperbolic equation with logarithmic nonlinearity. Nonlinear Anal. 184, 239-257 (2019)

18. Lian, W., Xu, R.: Global well-posedness of nonlinear wave equation with weak and strong damping terms and logarithmic source term. Adv. Nonlinear Anal. 9, 613-632 (2020)

19. Lions, J.L:: Quelques Méthodes de Résolution des Problèmes aux Limites Non Linéaires. Dunod, Paris (1969)

20. Liu, W.J., Yu, J.: On decay and blow-up of the solution for a viscoelastic wave equation with boundary damping and source terms. Nonlinear Anal. 74, 2175-2190 (2011)

21. Liu, Y.: On potential wells and applications to semilinear hyperbolic equations and parabolic equations. Nonlinear Anal. 64, 2665-2687 (2006)

22. $\mathrm{Ma}$, L., Fang, Z.B.: Energy decay estimates and infinite blow-up phenomena for a strongly damped semilinear wave equation with logarithmic nonlinear source. Math. Methods Appl. Sci. 41, 2639-2653 (2018)

23. Messaoudi, S.A.: Blow up and global existence in a nonlinear viscoelastic wave equation. Math. Nachr. 260, 58-66 (2003)

24. Nicaise, S., Pignotti, C.: Stability and instability results of the wave equation with a delay term in the boundary or internal feedbacks. SIAM J. Control Optim. 45, 1561-1585 (2006)

25. Nicaise, S., Pignotti, C.: Interior feedback stabilization of wave equations with time dependent delay. Electron. J. Differ Equ. 2011, 41 (2011)

26. Park, S.-H., Lee, M.J., Kang, J.-R.: Blow-up results for viscoelastic wave equations with weak damping. Appl. Math. Lett. 80, 20-26 (2018)

27. Park, S.H.: Energy decay for a von Karman equation with time-varying delay. Appl. Math. Lett. 55, 10-17 (2016)

28. Payne, L., Sattinger, D.: Saddle points and instability of nonlinear hyperbolic equation. Isr. J. Math. 226, 273-303 (1975)

29. Sattinger, D.H.: On global solution of nonlinear hyperbolic equations. Arch. Ration. Mech. Anal. 30, 148-172 (1968)

30. Vitillaro, E.: Global existence theorems for a class of evolution equations with dissipation. Arch. Ration. Mech. Anal. 49 155-182 (1999)

31. Wu, S.-T.: Blow-up of solution for a viscoelastic wave equation with delay. Acta Math. Sci. Ser. B Engl. Ed. 39, 329-338 (2019)

32. Yang, Z: Existence and energy decay of solutions for the Euler-Bernoulli viscoelastic equation with a delay. Z. Angew. Math. Phys. 66, 727-745 (2015)

\section{Submit your manuscript to a SpringerOpen ${ }^{\circ}$ journal and benefit from:}

- Convenient online submission

- Rigorous peer review

- Open access: articles freely available online

- High visibility within the field

- Retaining the copyright to your article

Submit your next manuscript at $\gg$ springeropen.com 